\title{
Development and Testing: Cervical Cancer Prevention Questionnaire Based on Theory of Planned Behavior in Chile
}

\author{
María-Teresa Urrutia ${ }^{* *}$ and Oslando Padilla ${ }^{2}$ \\ ${ }^{1}$ Nurse-Midwifery, Research Professor and Director Doctorate in Nursing Science, Faculty of Nursing, Universidad Andrés Bello, Chile \\ ${ }^{2}$ Statistician, Public Health Department, School of Medicine, Pontificia Universidad Católica de Chile \\ ${ }^{\star}$ Corresponding author: Maria Teresa Urrutia Soto, Research Professor, Director Doctorate in Nursing Science, Faculty of Nursing, Universidad Andres Bello, Chile
}

Received: January 11, 2021; Accepted: January 18, 2021; Published: January 27, 2021

\begin{abstract}
The purpose of this study was to developmentally and psychometrically validate the cervical cancer prevention questionnaire (CPCC-16) based on Theory of Planned Behavior in Chilean women. The patient sample was 967 women. Confirmatory factor analysis was used to evaluate factor structure, Cronbach's alpha for internal consistency and t-test for criteria validity. The development and validation of the questionnaire resulted in six factors with 16 items, demonstrating a bi-factorial structure. Cronbach's alpha was higher than 80 in the questionnaire and its factors. To generate a valid and reliable questionnaire that measures, under a theory of behavior, more than one preventive behavior in cervical cancer is an important advancement that fills a gap in nursing research.
\end{abstract}

Keywords: Cervical cancer, Prevention, Instrument development

Precis: The CPCC-16 questionnaire is a validated and reliable instrument, with 16 items distributed in a bi-factorial structure, useful for clinical and research area.

Call Outs: Behaviors are the principal causes of deaths from cancer, and thus, a reliable and validated questionnaire is necessary to measure these behaviors (before method).

The validated and reliable questionnaire will be useful to measure cervical cancer preventive behaviors as a whole, but it will also be useful to identify theory constructs (after method).

The new questionnaire will be useful to measure more than one preventive behavior in cervical cancer; therefore, it fills a gap in clinical and research area (after discussion).

\section{Introduction}

Theory of Planned Behavior (TPB) has been a framework to explain and predict behaviors [1], and its ability as a framework intervention has been supported by previous studies [2-4]. TPB postulates that the motivations of people to change are based on their perceptions of norms, attitudes, and control over behaviors, and each of these factors can either increase or decrease the intention to change their behavior. The intention to change behavior is directly related to behavioral change [5,6]. Cervical Cancer (CC) prevention has been one topic that has been studied under this theory [7-11].

There are two methods to stop CC: to prevent its pre-cancer and to identify and treat the cancer before it becomes a true cancer $[12,13]$. The first method includes behaviors, including the use of condoms during sex, limiting the number of sexual partners, not smoking and obtaining the Human Papilloma Virus (HPV) vaccine; the second method includes having regular screenings $[13,14]$. The CC prevention questionnaire (CPCC-16) was developed based on TPB to measure CC preventative behaviors.

\section{Background}

Even where screening is widely available and methods to prevent CC are known, there is an important barrier in adopting these methods by women. Thus, understanding the factors that affect preventive behavior remains an important issue.

TPB has been previously used by several studies to understand how cervical cancer preventive behaviors are carried out, and the intent to perform the behavior is explained $[8,15,16]$. The main behaviors studied are those related to the detection of CC, such as adherence to the HPV $[8,11,17]$ tests $[7,10,15,18,19]$. Some studies have described the use of TBP and HPV vaccination intentions $[16,20]$. The use of condoms has been studied; however, these studies are not always related to CC prevention and are mainly examined in an adolescent population $[21,22]$. To the best of our knowledge, there have been no previous studies using questionnaires to measure more than one preventive behavior using TPB as a framework.

Regarding the psychometric properties of the questionnaires used in CC prevention, the reliability and/or validity of the instruments has not always been reported $[10,11,16,20,21]$, or they have been incompletely reported [7-9,17,19]. Research on TPB with other behaviors indicates the same problem $[2,3,23]$. The author and creator 
of TPB [1] supports these findings, describing the measures of the theory constructs as fallible with respect to reliability and construct validity, and thus, it is difficult to test the theory.

With regard to TBP as a framework, the literature indicates that the most important theory construct studied has been intention [24] and that some research studies have only partially studied the TBP components [15].

Behaviors are the principal causes of deaths from cancer, and infections such as HPV are responsible for up to $25 \%$ of cancer cases in low and middle-income countries [25]. If the solid foundation of TBP and the relevance to prevent CC worldwide are considered, then a questionnaire that is reliable and valid, which permits the ability to simultaneously measure more than one CC preventive behavior and to test the four-principal construct of TPB, may be useful in different countries and contexts. Thus, it is useful to have a CC prevention questionnaire based on TBP.

The purpose of this study was to develop and psychometrically validate a new questionnaire based on Theory Planned Behavior (TPB) with relation to Cervical Cancer (CC) prevention (known as the CPCC-16 questionnaire).

\section{Method}

This study is a part of a larger cross-sectional study about Social Determinants related to the adherence to CC screening (FONDECYT \#11130626); this article focuses on the development and testing of one of the questionnaires used in the project, which was performed in two phases: scale development and psychometric evaluation.

\section{Sample/Participants}

This research study was performed on a total of 967 Chilean females, between 25 to 64 years old, under Chilean national public health care coverage (known as FONASA); these participants attended four primary health care centers in the Servicio de Salud Metropolitano Sur-Oriente (Southeast Metropolitan Public Health Service) in Santiago, Chile. The sample size was calculated according to the larger study aims considering an effect size of 0.1 , power analysis of $80 \%, 15$ latent variables and 40 observed, and a significance level of $95 \%$. The sample was obtained according to the recommendations related to the questionnaire validation [26-28]. The exclusion criteria included having had a hysterectomy and CC disease. Females who had agreed to participate were randomly selected and recruited by telephone between March 2014 and October 2015.

\section{Scale Development}

The questionnaire was developed based on TPB and according to Icek Ajzen's recommendations [29]. The first step was to define the behavior; therefore, four behaviors were included: annual gynecological check, updated Papanicolaou test (Pap), condom use on sexual relations and having a single partner (at the same time). Behaviors related to the HPV vaccine or HPV screenings were not considered because they are not available in the public health care system where the study was performed. Figure 1 shows the construct and preventive behaviors considered in the questionnaire. The second

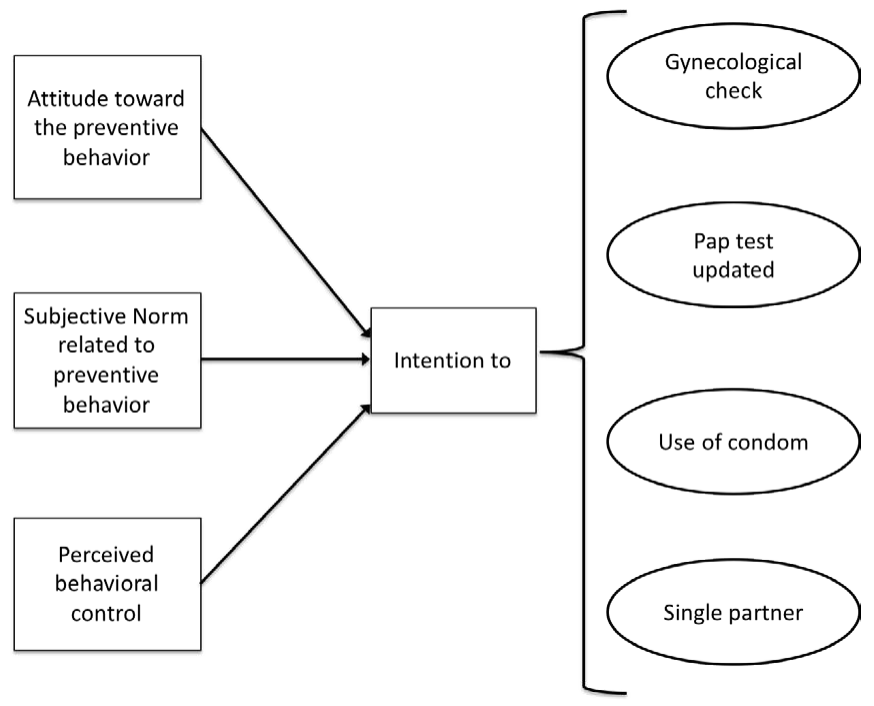

Figure 1: Theory of Planned Behavior Constructs and Cervical Cancer Preventive Behaviors considered in the Original Questionnaire.

step was defining the population; females between 25 to 64 years old were selected because they are the target group for cervical cancer screening and prevention interventions in Chile. The third step was formulating items; they were developed to assess the major constructs of TPB for each preventive behavior selected in the first step: attitude, subjective norm, perceived behavioral control, and intention. The items were given feedback from content experts and then pilot tested on ten females from the target population. The questionnaire was developed in the Spanish language and back-translated for this article.

\section{Psychometric Evaluation}

Construct validity was performed by Confirmatory Factor Analysis (CFA), and reliability was assessed using ordinal Cronbach's alpha. Three models were adjusted: one model with the four TBP constructs, the second with a bifactorial model considering the four TBP constructs and the four CC prevention behaviors, and the last model considered the four constructs from TBP; four behaviors but three of them were grouped in one factor. Diagonally Weighted Least Squares (DWLS) were used to estimate the models because the variables were measured using the four-point ordinal scale. The fit model was evaluated using normed chi-squared (chi-squared/degree of freedom) with two comparative fit indices: Comparative Fit Index (CFI) and Tucker-Lewis Index (TLI); Root Mean Square Error of Approximation (RMSEA) was used as parsimonious fit indices. We considered CFI and TLI values $>0.95$, with RMSEA $<0.05$ as good; CFI and TLI values between 0.90-0.95 and RMSEA between 0.05-0.08 were acceptable; and CFI and TLI values $<0.90$ or RMSEA $>0.08$ were unacceptable. The statistical significance of each item as it is related to the factor, as well as whether an item shared a common conceptual meaning with the factor, was also considered. The PAP test and gynecological check status were used as external criteria to validate the questionnaire. The scores of each factors of the questionnaire were calculated by regression method, using standardized variables and the factor scores between women with adherence to CC screening and without adherence or with annual gynecological check and without 
it were compared using t-Student test for independent samples. Data were analyzed using R Statistical Program and the lavaan package.

\section{Instrument}

The proposed instrument (Appendix 1) consisted of 16 items related to CC prevention behaviors, which were divided into 4 dimensions according to the TPB constructs: attitudes (4 items), subjective norms (4 items), perceived behaviors control (4 items), and intentions (4 items). Each item was evaluated using a Likert scale of four alternatives (strongly agree/very good=1 to strongly disagree/ very bad $=4$ ). Such a scale is used to force directionality of a response (de Vellis, 200 [30]) in a population where culture (Hispanic) tends to avoid conflict, resulting in a frequent selection of neutral alternatives (Antshel, $2002[31])$.

\section{Ethical Considerations}

This study was approved by the University of the Principal Investigator and by the health care service to which the women belonged. Written informed consent was obtained by all of the

Table 1: Characteristics of the women $(n=967)$.

\begin{tabular}{|l|c|}
\hline Characteristic & Value \\
\hline Annual Gynecological Check, $\mathrm{n}(\%)$ & $537(55.5)$ \\
\hline Pap test in the last three years, $\mathrm{n}(\%)$ & $740(76.5)$ \\
\hline Have a partner, $\mathrm{n}(\%)$ & $766(79.2)$ \\
\hline Number of partners, mean \pm SD (range) & $2.69 \pm 2.73(1$ to 40$)$ \\
\hline Use of Condom, $\mathrm{n}(\%)$ & $65(6.7)$ \\
\hline Always & $85(8.8)$ \\
\hline Almost always & $102(10.5)$ \\
\hline Never always & $715(73.2)$ \\
\hline Never & \\
\hline
\end{tabular}

Table 2: Fit Statistics for the three models calculated $(n=967)$.

\begin{tabular}{|l|c|c|c|c|}
\hline Factor Model & $\chi^{2} / \mathbf{d f}$ & CFI & TLI & RMSEA (CI 95\%) \\
\hline Four Factor & 52.75 & 0.909 & 0.888 & $0.231(0.226-0.237)$ \\
\hline Eight Factor (bifactorial) & 1.77 & 0.999 & 0.998 & $0.028(0.020-0.036)$ \\
\hline Six Factor (bifactorial) & 1.94 & 0.999 & 0.998 & $0.031(0.024-0.038)$ \\
\hline
\end{tabular}

participants. All of the questions that the women had about CC were answered after the interview.

\section{Results}

The mean age of the participants was $43.37 \pm 10.77$ years, with the mean educational level being $10.97 \pm 3.4$ years. The CC preventive behaviors of the women are shown in Table 1 .

Three models were calculated to achieve the best fit with the data (Table 2). The first model considered the proposed questionnaire with four factors, but the goodness of fit was not good (TLI $<0.9$ and RMSEA=0.231); the modified indices suggested the inclusions of correlations between the items with similar wording; and the correlations between intention and subjective norms $(\mathrm{r}=0.863)$ and intention and perceived behaviors control $(r=0.939)$ were too high.

These results suggested the consideration of second-order models, which explain the high correlations between factors, but this approach did not resolve the problem related to the correlations between preventive behavioral items. Thus, a bifactorial model was tested as a plausible alternative; one side with four factors related to TPB and the other side with four factors related to CC preventive behaviors were considered; the four factors within each side were correlated but not between the sides. The second model showed a good fit but with two high correlations: one of the correlations between an annual gynecological check and single partner $(r=0.839)$ and the other between an annual gynecological check and updated PAP test $(\mathrm{r}=0.98)$. Thus, the decision was to place the correlations together into one factor. This model good fits the data, thus indicating a bi-factor structure with six factors, four of which were the TPB components and the other two were CC preventive behaviors. The results of external criteria validly of the questionnaire are in Table 3.

The new questionnaire (Appendix 1) called CPCC-16 (Conductas Preventivas en Cáncer Cérvicouterino-16 items/ Preventive Behaviors on Cervical Cancer -16 items) consisted of 16 items, which were distributed into six factors. The complete standardized parameters of the bifactorial model are shown in Figure 2. According to the bifactorial structure, each item corresponds to two factors. A summary of the CPCC-16 bifactorial model with factors, number of items and Cronbach's alpha are shown in Table 4.

Table 3: External criterion validity through comparison between groups for Papanicolaou test and Gynecological check.

\begin{tabular}{|c|c|c|c|c|c|c|}
\hline \multirow{3}{*}{ Factor } & \multicolumn{3}{|c|}{ Papanicolaou Test in the last three years } & \multicolumn{3}{|c|}{ Anual Gynecological Check } \\
\hline & Yes & No & & Yes & No & \\
\hline & Mean (SD) & Mean (SD) & P value $\left({ }^{a}\right)$ & Mean (SD) & Mean (SD) & $P$ value $\left({ }^{a}\right)$ \\
\hline 1. Cervical cancer preventive behaviors & $-0.06(0.39)$ & $-0.16(0.41)$ & $<0.001$ & $-0.05(0.38)$ & $-0.12(0.41)$ & 0.004 \\
\hline 2. Condom use as cervical cancer prevention & $-0.03(0.63)$ & $-0.05(0.63)$ & 0.631 & $-0.03(0.64)$ & $-0.04(0.63)$ & 0.876 \\
\hline 3. Attitude to cervical cancer prevention & $-0.00(-35)$ & $-0.05(0.38)$ & .045 & $-0.02(0.35)$ & $-0.01(0.36)$ & .738 \\
\hline 4. Perceived norm & $-0.01(0.40)$ & -0.11 .46 & .002 & $-0.02(0.42$ & $-0.06(0.42)$ & .152 \\
\hline 5. Perceived behaviors control & $-0.00(0.43)$ & $-0.29(0.65)$ & $<0.001$ & $.03(0.39)$ & $-0.19(0.60)$ & $<0.001$ \\
\hline 6. Intention & $-0.01(0.26)$ & $-0.11(0.30)$ & $<0.001$ & $-0.00(0.25)$ & $-0.07(0.30)$ & $<0.001$ \\
\hline
\end{tabular}

(a) T-Student was used to compare group values. 


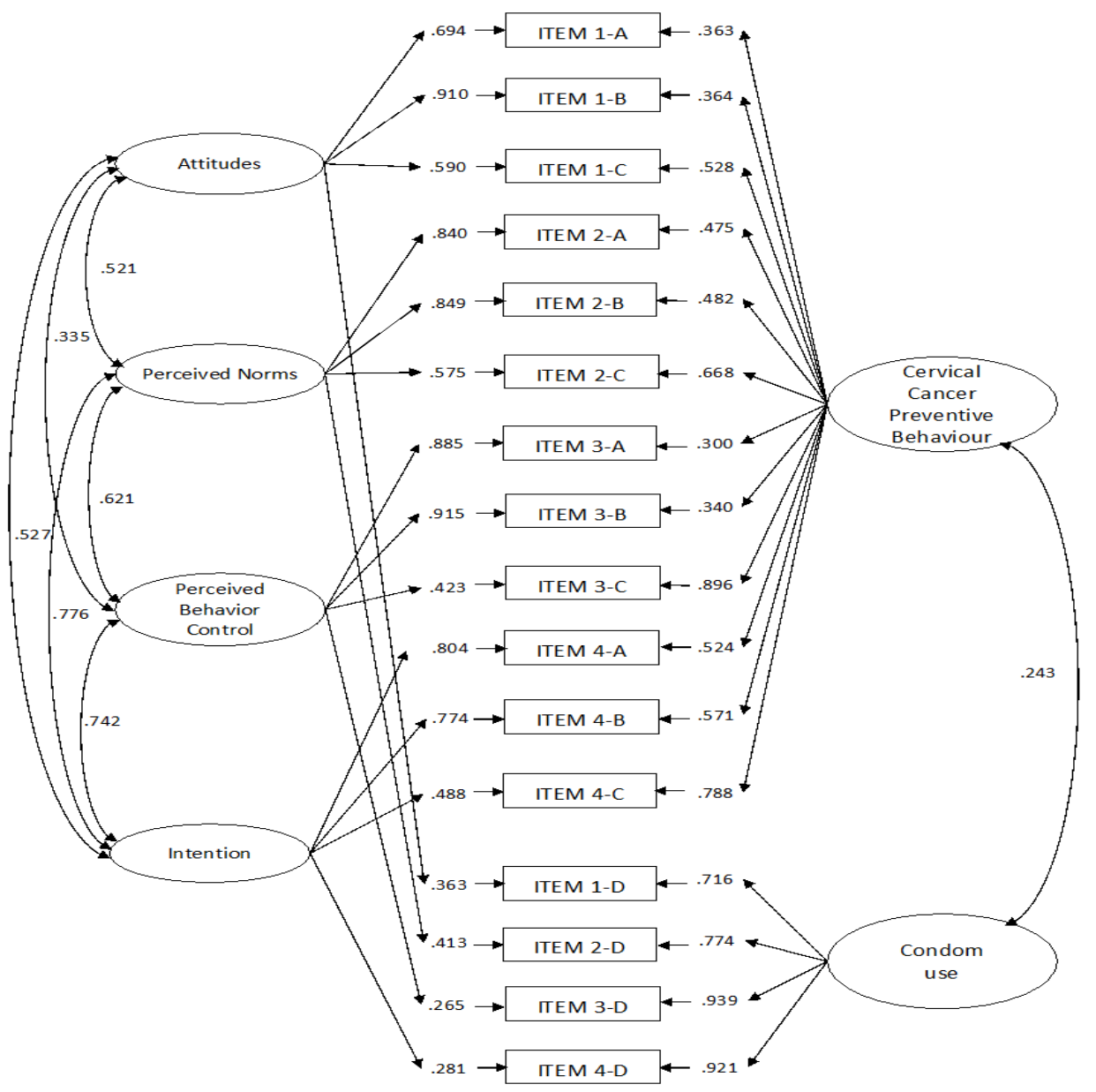

Figure 2: Complete Standardized Parameters for the Bifactor Model of CPCC-16 ( $\mathrm{n}=967)$.

Table 4: Name of factor, items and Cronbach's alpha $(n=967)$.

\begin{tabular}{|l|c|c|}
\hline Factor & No of items & Cronbach's alpha \\
\hline 1. Cervical cancer preventive behaviors & 12 items & 0.95 \\
\hline 2. Condom use as cervical cancer prevention & 4 items & 0.93 \\
\hline 3. Attitude to cervical cancer prevention & 4 items & 0.81 \\
\hline 4. Perceived norm & 4 items & 0.87 \\
\hline 5. Perceived behaviors control & 4 items & 0.81 \\
\hline 6. Intention & 4 items & 0.86 \\
\hline CPCC-16 Questionnaire & & 0.94 \\
\hline
\end{tabular}

\section{Discussion}

The first considerations to note are how the structure of the original questionnaire, without varying the number of items, was shown throughout the analysis. The initial questionnaire was created considering the underpinning of TPB constructs, where four factors were proposed. However, a questionnaire where only the theory constructs are considered was unacceptable, and thus, it was necessary to include the behavioral dimensions. However, although the second tested model with eight factors has good fit, it should not be considered a final model because it has two dimensions highly correlated (Pap test and Gynecological check). Thus, the result was a model with six dimensions in which all of the factors loading were significant, although some of the factors exhibited values lower than 0.4 .
The final questionnaire was very consistent with another underpinning construct, that was not considered from the beginning (CC preventive behaviors). This result allows us to extend its usefulness, not only to test the TPB but also to analyze and explain CC preventive behaviors using this theoretical model.

The criteria validity shows that the final questionnaire is useful to associate the TPB constructs with the behaviors. The women with different CC screening or gynecological check behaviors did not show differences in condom factor scores; explanations could be because the condom use is not associated with cervical cancer prevention $[32,33]$, or because the use of condom could be overestimated in the sample since it is an expected social behavior.

There are many contexts where the new questionnaire could be useful and where the TBP has demonstrated its utility: to assess the acceptability of preventive behaviors as a whole among the target group of women currently engaged in preventive programs $[8,11]$, to evaluate the evolution of the intention across time $[8,17]$, to evaluate the strategies of cervical cancer prevention programs $[8,11,20]$ and to determine the factors that influence the behaviors $[7,9,10,15,18,20]$. CC remains a relevant problem, particularly in underdeveloped countries and ethnic minorities in developed countries [10]. Thus, instruments related to the prevention of CC could be very useful in many contexts. Theoretically based models of behaviors are also useful and necessary for the development of effective interventions $[2,9,16,34]$. 
A second consideration, related to the results, is why three preventive behaviors proposed in the initial questionnaire were collapsed into 1 factor, CC preventive behaviors (annual gynecological check, updated PAP test and to have a single partner). The second model tested demonstrated that each of the four behaviors proposed as a factor, but the results suggested other structures. This finding could be explained by it being necessary to consider who participated in the behavior and who decided it. Therefore, CC preventive behavior factor focuses on behaviors in which the decision is primarily or only related with females, and the condom use factor indicates a behavior in which consensus is required between a woman and her partner.

Other explanations to account for the second consideration and indirectly related to the previous consideration could be that the CC preventive factor included behaviors that are not clearly associated with sexual life, and thus, it is not necessary to have an active sexual life. This is in contrast to the second factor where having sexual intercourse is the principal focus. The association between HPV and $\mathrm{CC}$ is one of the strongest described [13], but the relationship between sexual behaviors and CC risk has not been previously recognized by the women [35]. The use of a condom as CC preventive behavior was described in only $5.6 \%$ of the women [32], and in a Chilean study [33], only $27.6 \%$ of the women described sexual intercourse as a risk factor of CC. Thus, these reasons could explain the way that the preventive behaviors were grouped.

Related to factor loading of the items, there are only two items (items 3D and 4D), which have values less than 0.3 , that could indicate that both items may be explained by the condom use factor rather than the intention and perceived behavioral control factors. To the best of our knowledge, there are no studies in which the results fit into a bifactorial model for preventive behaviors and TBP. This may be due to the focus of the research study on only one behavior and not as a group. There are many diseases that can be prevented by practicing some behavior, and the TPB is a solid theory that can help with its understanding. Thus, the CPCC-16 could be considered an example of how more than one behavior could be studied under a bifactorial structure under this theory.

One limitation of this research is that the new questionnaire did not include all of the CC preventive behaviors recognized in the literature, so it could be useful to develop a new version by adding these behaviors. However, it is important to consider that to include other behaviors; the questionnaire could require that the age range of the population be broader where the questionnaire will be used, because preventive behaviors, such as HPV vaccination, are targeted at a younger population where the decision does not often depend on them alone.

\section{Conclusion}

The new questionnaire is a contribution to the measurement of preventive behaviors in cervical cancer, enabling its use in research and a clinical setting. The use of TPB as a framework of this questionnaire and the structure shown by the questionnaire are important contributions to advance the cervical cancer arena because the new questionnaire will not only be useful to measure cervical cancer preventive behaviors as a whole but also identify the theory constructs. To have a valid and reliable questionnaire that measures, under a theory of behavior, more than one preventive behavior in cervical cancer is an important advance because it fills a gap in clinical and research area.

\section{References}

1. Ajzen I (2015) The theory of planned behaviour is alive and well, and not ready to retire: a commentary on Sniehotta, Presseau, and Araujo-Soares. Health Psychology Review 9: 131-137. [crossref]

2. Armitage CJ, Talibudeen L (2010) Test of a brief theory of planned behaviour-based intervention to promote adolescent safe sex intentions. British Journal of Psychology 101: 155-172. [crossref]

3. Booth AR, Norman P, Goyder E, Harris PR, Campbell MJ (2014) Pilot study of a brief intervention based on the theory of planned behaviour and self-identity to increase chlamydia testing among young people living in deprived areas. British Journal of Health Psychology 19: 636-651. [crossref]

4. Steinmetz H, Knappstein M, Ajzen I, Schmidt P, Kabst R (2016) How Effective are Behavior Change Interventions Based on the Theory of Planned Behavior? Zeitschrift für Psychologie 224: 216-233.

5. Edberg M (2015) Individual health behavior theories. In M. Edberg (Ed.), Essentials of Health Behavior.Social and Behavioral Theory in Public Health. Burlington: Jones \& Bartlett Learning.

6. Grim M, Hortz B (2016) Theory in health promotion programs. In C. Fertman \& D. Allensworth (Eds.), Health promotions programs. From Theory to Practice (second ed., pp. 55-56). San Francisco: Jossey-Bass.

7. Jalilian F, Emdadi S (2012) Factors Related to Regular Undergoing Pap-smear Test: Application of Theory of Planned Behavior. Journal of Research in Health Sciences 11: 103-108. [crossref]

8. Ogilvie GS, Smith LW, van Niekerk D, Khurshed F, Pedersen HN, et al. (2016) Correlates of women's intentions to be screened for human papillomavirus for cervical cancer screening with an extended interval. BMC Public Health 16: 213.

9. Roncancio AM, Ward KK, Fernandez ME (2013) Understanding cervical cancer screening intentions among Latinas using an expanded theory of planned behavior model. Behavioral Medicine 39: 66-72. [crossref]

10. Roncancio AM, Ward KK, Sanchez IA, Cano MA, Byrd TL, et al. (2015) Using the Theory of Planned Behavior to Understand Cervical Cancer Screening Among Latinas. Health Education and Behavior 42: 621-626. [crossref]

11. Smith L, Khurshed F, van Niekerk D, Krajden M, Greene S, et al. (2014) Women's intentions to self-collect samples for human papillomavirus testing in an organized cervical cancer screening program. BMC Public Health 14: 2-9. [crossref]

12. American Cancer Society. (2016, December 5, 2016). Can Cervical Cancer Be Prevented? Retrieved from

13. Shepherd JP, Frampton GK, Harris P (2011) Interventions for encouraging sexual behaviours intended to prevent cervical cancer. Cochrane Database of Systematic Reviews 4: CD001035. [crossref]

14. Centers for disease control and prevention. (2017, February 9, 2017). What Can I Do to Reduce My Risk of Cervical Cancer? Retrieved from

15. Chen S-L, Tsai S-F, Hsieh M-M, Lee L-L, Tzeng Y-L (2016) Factors Predicting Nurse Intent and Status Regarding Pap Smear Examination in Taiwan: a Cross-sectional Survey. Asian Pacific Journal of Cancer Prevention 17: 165-170. [crossref]

16. Gerend MA, Shepherd JE (2012) Predicting human papillomavirus vaccine uptake in young adult women: comparing the health belief model and theory of planned behavior. Annals of Behavioral Medicine 44: 171-180. [crossref]

17. Ogilvie GS, Smith LW, van Niekerk DJ, Khurshed F, Krajden M, et al. (2013) Women's intentions to receive cervical cancer screening with primary human papillomavirus testing. International Journal of Cancer 133: 2934-2943. [crossref]

18. Kim H (2014) Awareness of Pap testing and factors associated with intent to undergo Pap testing by level of sexual experience in unmarried university students in Korea: results from an online survey. BMC Women's Health 14: 2-13. [crossref] 
19. Sandberg T, Conner M (2009) A mere measurement effect for anticipated regret: impacts on cervical screening attendance. The British Journal of Social Psychology 48: 221-236. [crossref]

20. Bennett K, Buchanan J, Adams A (2012) Social-Cognitive Predictors of Intention to Vaccinate Against the Human Papillomavirus in College-Age Women. The Journal of Social Psychology 152: 480-492.

21. Espada JP, Morales A, Guillen-Riquelme A, Ballester R, Orgiles M (2016) Predicting condom use in adolescents: a test of three socio-cognitive models using a structural equation modeling approach. BMC Public Health 16: 35.

22. Jemmott JB, Hennessy M (2012) The Reasoned Action Approach in HIV RiskReduction Strategies for Adolescents. The ANNALS of the American Academy of Political and Social Science 640: 150-172.

23. Noonan D, Kulbok P, Yan G (2011) Intention to smoke tobacco using a waterpipe among students in a southeastern U.S. College. Public Health Nursing 28: 494-502. [crossref]

24. Exum ML, Turner MG, \& Hartman JL (2011) Self-Reported Intentions to Offend: All Talk and No Action? American Journal of Criminal Justice 37: 523-543.

25. World Health Organization. (2017, February 17). Cancer. Retrieved from

26. Nunnaly J, Bernstein I (1994a) The assessment of reliability. In Nunnaly J \& Bernstein I (Eds.), Psychometric theory: McGraw Hill.

27. Nunnaly J, Bernstein I (1994b) Exploratory factor analysis II: Rotation and other topics. In Nunnaly J \& Bernstein I (Eds.), Psychomethic theory: McGraw Hill.
28. Pett M, Lackey N, Sullivan J (2003) Assesing the characteristics of matrices. In Pett M, Lackey N, \& Sullivan J (Eds.), Making sense of factor analysis. The use of factor analysis for instrument development in health care research: housand Oaks: Sage publications.

29. Ajzen I (2006) Constructing Theory of Planned Behavior Questionnaire Retrieved from

30. de Vellis R (2003) Guidelines in scale development. In de Vellis R (Ed.), Scale development: Theory and applications (pp. 60-101): Thousand Oaks: Sage Publications

31. Antshel K (2002) Integrating culture as a means of improving treatment adherence in the Latino population. Psychology, Health \& Medicine 7: 435-449.

32. Raychaudhuri S, Mandal S (2012) Socio-Demographic and Behavioural Risk Factors for Cervical Cancer and Knowledge, Attitude and Practice in Rural and Urban Areas of North Bengal, India. Asian Pacific Journal of Cancer Prevention 13: 1093-1096. [crossref]

33. Urrutia M (2012) Creencias sobre Papanicolaou y cáncer cérvicouterino en un grupo de mujeres chilenas. Revista Chilena de Obstetricia y Ginecología 77: 3-10.

34. Luszczynska A, Durawa A, Scholz U, Konll N (2012) Empowerment Beliefs and Intention to Uptake Cervical Cancer Screening: Three Psychosocial Mediating Mechanisms. Women and Health 52: 162-181. [crossref]

35. Opoku CA, Browne ENL, Spangenberg K, Moyer C, Kolbilla D, et al. (2016) Perception and risk factors for cervical cancer among women in northern Ghana. Ghana Medical Journal 50: 84. [crossref]

\section{Citation:}

Urrutia MT, Padilla O (2021) Development and Testing: Cervical Cancer Prevention Questionnaire Based on Theory of Planned Behavior in Chile. ARCH Women Health Care Volume 4(1): 1-7. 


\section{Appendix 1}

Instructions: The following phrases are some ideas about behaviors. Mark your level of agreement with a cross for each phrase. There are no right or wrong answers, so if you are not sure about some questions or do not know an answer, feel free to answer with what you think.

\begin{tabular}{|c|c|c|c|c|c|}
\hline \multicolumn{6}{|c|}{ 1. How do you evaluate each of the following behaviors: } \\
\hline & & Very good & Good & $\mathrm{Bad}$ & Very Bad \\
\hline 1.A & Have a gynecological check (with a nurse midwife or gynecologist) annually & & & & \\
\hline $1 . \mathrm{B}$ & Take the PAP test when appropriate. & & & & \\
\hline 1.C & Have a single sexual partner (at the same time) & & & & \\
\hline 1.D & Use condoms in (all) sexual relationships. & & & & \\
\hline \multicolumn{6}{|c|}{ 2. Most people who are important to me would agree to: } \\
\hline & & Strongly Agree & Agree & Disagree & Strongly Disagree \\
\hline 2.A & Have a gynecological check (with a nurse midwife or gynecologist) every year & & & & \\
\hline 2.B & Take the PAP test when appropriate. & & & & \\
\hline 2.C & Have a single sexual partner (at the same time) & & & & \\
\hline 2.D & Use condoms in (all) sexual relationships. & & & & \\
\hline \multicolumn{6}{|c|}{ 3. I am confident that I can: } \\
\hline & & Strongly Agree & Agree & Disagree & Strongly Disagree \\
\hline 3.A & Have a gynecological check (with a nurse midwife or gynecologist) every year & & & & \\
\hline $3 . \mathrm{B}$ & Take PAP when appropriate. & & & & \\
\hline 3.C & Have a single sexual partner (at the same time) & & & & \\
\hline 3.D & Use condoms in (all) sexual relationships. & & & & \\
\hline \multicolumn{6}{|c|}{ 4. In the future I want to: } \\
\hline & & Strongly Agree & Agree & Disagree & Strongly Disagree \\
\hline 4.A & Have a gynecological check (with a nurse midwife or gynecologist) every year & & & & \\
\hline $4 . \mathrm{B}$ & Take PAP when appropriate. & & & & \\
\hline 4.C & Have a single sexual partner (at the same time) & & & & \\
\hline 4.D & Use condoms in (all) sexual relationships. & & & & \\
\hline
\end{tabular}

